\title{
Avaliação de Desempenho de Redes Veiculares em Condições Adversas
}

\author{
Willian Becker de Souza, Joilson Alves Junior, Danilo R. de Assis, Emilio C.G. Wille e Tania L. Monteiro
}

Resumo-As Redes $\mathrm{Ad}$ Hoc Veiculares (VANETs) fazem parte dos Sistemas Inteligentes de Transporte (ITS) e foram desenvolvidas para possibilitar a comunicação entre os veículos. A maioria das pesquisas em VANETs utilizam simuladores, os quais permitem a avaliação rápida e barata de protocolos e aplicações. Para tornar os resultados das análises mais significativos, os cenários das simulações devem refletir o mundo real, incluindo ambientes com condições adversas (aquelas prejudiciais para o bom funcionamento da rede). Portanto, este trabalho propõe realizar avaliações de desempenho, por meio de simulações, em redes veiculares com condições adversas. Experimentos de simulação comprovam que o conjunto AODV/UDP apresenta os melhores resultados para a taxa de entrega na maioria das condições adversas analisadas. Entretanto, quando o TCP está presente na rede, o protocolo DSR apresenta as melhores taxas.

Palavras-Chave - VANETs, Avaliação, Condições Adversas, Simulações, Protocolos.

\section{INTRODUÇÃO}

O crescimento do número de veículos automotores nos últimos anos passou a exigir novas maneiras de gerenciar o tráfego. Utilizando diferentes tecnologias, os sistemas inteligentes de transporte (ITS) visam gerenciar o tráfego de forma inteligente (monitorando e regulando as ações de trânsito automaticamente). Os ITS fornecem diversos serviços inovadores, entre eles destacam-se: a transmissão dos dados sobre as condições do tráfego veicular em tempo real e o gerenciamento da mobilidade urbana [1][2].

As Redes $A d H o c$ Veiculares (VANETs) são uma parte importante dos ITS e foram desenvolvidas principalmente para melhorar a segurança e o conforto dos motoristas e passageiros. Estas redes possibilitam que os veículos troquem diferentes tipos de informações, as quais podem variar de mensagens de entretenimento a alertas de segurança. Como esses sistemas geralmente consistem em muitos veículos, testes no mundo real são muito custosos (caros e demorados). Portanto, a maioria das pesquisas com VANETs utilizam simuladores, os quais permitem avaliações rápidas e baratas de protocolos e aplicações. Como os estudos de simulações são usados para avaliar a viabilidade das redes no mundo real, devem sempre refletir as condições reais. Porém, como as simulações são controláveis pelo usuário, geralmente consideram ambientes favoráveis para o funcionamento das VANETs, ou seja, não consideram ambientes com condições prejudiciais para o bom funcionamento da rede (adversas), as quais também são frequentes nas comunicações veiculares [3][4].

Devido ao grande potencial dos ITS, principalmente no que tange a segurança durante a condução veicular, nos últimos anos, as redes veiculares têm sido alvo de pesquisas tanto na área acadêmica como na indústria automobilística [5].

Willian Becker de Souza, Joilson Alves Junior, Danilo R, de Assis, Emilio.C.G. Wille, Tania L Monteiro. Universidade Tecnológica Federal do Paraná(UTFPR), Av. Sete de Setembro 3165, 80230-901, Curitiba (PR), Brazil. E-mail: beckerwillian@hotmail.com,_joilson@utfpr.edu.br, daniloassis@utfpr.edu.br, ewille@utfpr.edu.br, tmonteiro@utfpr.edu.br.
Entretanto, para que estas pesquisas possam avançar, inicialmente é necessário entender e superar alguns problemas básicos que podem afetar a eficiência das comunicações. Um destes problemas é uma rede em condição adversa, isto é, uma rede fora da normalidade e desfavorável para o seu funcionamento. Conhecer e avaliar o desempenho das redes nestas condições, pode influenciar na escolha dos protocolos e aplicações mais adequados para maximizar o seu potencial. Além disso, os resultados das avaliações podem apresentar novos desafios para os projetistas de protocolos, assim como contribuir para o desenvolvimento de uma infraestrutura de comunicação veicular mais eficiente.

Portanto, esse trabalho tem como objetivo avaliar, por meio de simulações, os protocolos de roteamento $\mathrm{Ad}-\mathrm{Hoc} \mathrm{On}$ Demand Distance Vector (AODV) [6], Ad-Hoc On-Demand Multipath Distance Vector (AOMDV) [7], Dynamic Source Routing (DSR) [8] e Destination-Sequenced Distance-Vector Routing (DSDV) [9], em conjunto com os protocolos de transporte User Datagram Protocol (UDP) [10] e Transmission Control Protocol (TCP) [10], e a aplicação geradora de tráfego CBR. Para as avaliações foram criados diversos cenários adversos, os quais são: veículos enviando até 256 mensagens/s; veículos enviando mensagens com até 4096 bytes de tamanho; ambientes com alta densidade (até 400 veículos por $\mathrm{km}^{2}$ ); ambientes com alta velocidade (até $150 \mathrm{~km} / \mathrm{h}$ ).

O restante deste trabalho está organizado da seguinte forma: a Seção II exibe os trabalhos relacionados; a Seção III mostra as redes veiculares em condições adversas; a Seção IV apresenta a avaliação de desempenho; a Seção V apresenta os resultados; e por fim a Seção VI apresenta a conclusão.

\section{TRABALHOS RELACIONADOS}

$\mathrm{Na}$ literatura atual é possível encontrar diversos trabalhos que abordaram análises de desempenho em VANETs

Em [11] foi utilizado o NS-3 e o BonnMotion para mostrar uma avaliação dos protocolos de roteamento AODV, OLSR e DSDV para diferentes, velocidades e densidades. As métricas analisadas foram: taxa de entrega, atraso e vazão. Com relação às características das simulações, foi utilizado um cenário de $2.000 \mathrm{~m}$ por $2.000 \mathrm{~m}$ com 40,50 e 60 nós com velocidades que variaram de $15 \mathrm{~m} / \mathrm{s}$ até $55 \mathrm{~m} / \mathrm{s}$. Os resultados mostraram que o DSDV e OLSR são melhores que AODV em cenários com poucos nós e baixa velocidade. Já em cenários mais densos e com maior velocidade, o OLSR apresenta um desempenho melhor que o AODV e DSDV.

Os autores em [12] apresentaram uma análise de desempenho dos protocolos de roteamento AODV, DSDV e DSR utilizando os simuladores SUMO e o NS-3. Neste estudo foram configuradas diferentes velocidades para os nós, 
considerou-se $30 \mathrm{~km} / \mathrm{h}$ para simular o tráfego de veículos nas cidades, e $100 \mathrm{~km} / \mathrm{h}$ para simular o tráfego de veículos nas rodovias, e um total de 30 nós na rede. Com relação aos parâmetros das simulações, foram utilizados tanto tráfego TCP quanto UDP. Como resultado, obteve-se que o AODV apresenta o melhor desempenho, adaptando-se bem a operação com nós em altas velocidades.

Com o uso do simulador NS-3, o trabalho em [13] testou diferentes modelos de propagação para avaliar o desempenho dos protocolos OLSR, DSDV, AODV e CLWPR quanto a taxa de entrega, atraso, vazão e jitter. Para as simulações, foi utilizada uma área de 1.000 por 1.000 metros, com 10, 20 ou 50 nós e uma velocidade média de $20 \mathrm{~m} / \mathrm{s}$. Os autores concluíram que na maioria dos cenários avaliados os protocolos CLWPR, DSDV e OLSR (nesta ordem), apresentaram os melhores desempenhos.

Utilizando o simulador de rede NS-2, o estudo apresentado em [14] apresentou uma comparação de desempenho entre os protocolos de roteamento AODV, DSDV e LSGR. As métricas utilizadas para a avaliação foram: taxa de entrega, sobrecarga de roteamento, atraso e vazão. Foram realizadas simulações em uma rede de $1.500 \mathrm{~m}$ por $1.200 \mathrm{~m}$, com 14 nós. Os resultados de simulação mostraram que o protocolo LSGR foi o que teve o maior destaque, apresentando melhor desempenho em relação aos seus concorrentes para as métricas atraso, vazão e taxa de entrega de pacotes.

Com os artigos citados percebe-se que existem vários estudos sobre avaliação e comparação de protocolos de roteamento para VANETs. Entretanto, o que torna o presente trabalho singular, são as análises e comparações de desempenho de protocolos de roteamento e protocolos de transporte em condições adversas (estes tipos de análises não foram realizadas anteriormente).

\section{REDES VEICULARES EM CONDIÇÕES ADVERSAS}

Condições Adversas são todos os fatores que podem prejudicar o funcionamento normal das redes veiculares, tornando maior a probabilidade de problemas ocorrerem.

\section{A. Problema}

O desenvolvimento e o controle das VANETs dependem da superação de diversos problemas ainda existentes. Problemas ainda pouco estudados são relativos às redes veiculares operando em condições adversas. As condições adversas mais comuns e consideradas neste trabalho são: veículos trafegando em velocidades muito altas; veículos transmitindo grandes volumes de dados; veículos transmitindo altas taxas de dados; veículos trafegando em ambientes com alta densidade.

Tais condições podem causar problemas como: perda de pacotes, desconexões, sobrecarga de roteamento, estouro de pilha, atraso, baixa vazão, conexões curtas e falta de tempo para transmitir o pacote todo. Entretanto, com o aumento da utilização dos sistemas inteligentes de transporte e com o advento da internet das coisas móveis, em um futuro próximo, todos os veículos necessitarão transmitir grandes volumes de dados com altas taxas de transmissão em ambientes com velocidades e densidades extremas. Por isso, todos estes cenários devem ser estudados e avaliados para que soluções possam ser propostas.

\section{AVALIAÇÃO DE DESEMPENHO DE REDES VEICULARES EM CONDIÇÕES ADVERSAS}

A avaliação de desempenho foi realizada utilizando um simulador de rede, o NS-2 [15], e um simulador de movimentos, o VanetMobisim [16], e consiste em avaliar métricas que revelem o desempenho da rede quando submetida a condições adversas.

\section{A. Métricas de desempenho}

Os sistemas, incluindo as VANETs, precisam ser avaliados com base em critérios que revelam o seu desempenho. Tais critérios são conhecidos como métricas de desempenho [17]. A métrica de desempenho utilizada neste trabalho é:

Taxa de Entrega (TE): é a proporção do número de pacotes recebidos no destino em relação ao número de pacotes enviados pela origem. Uma rede deve ter uma taxa de entrega alta a fim de ter um melhor desempenho e confiabilidade.

\section{B. Ambiente de simulação}

A rede criada para as simulações é uma área retangular de $1000 \mathrm{~m} \times 1000 \mathrm{~m}$. Todos os veículos que trafegam por esta rede transmitem seus sinais de radiofrequência em um raio de $250 \mathrm{~m}$ e se deslocam seguindo o modelo de movimentação estabelecido pelo VanetMobiSim. Além disso, o padrão de tráfego consiste de conexões com taxa de bits constante (CBR) considerando os protocolos de transporte UDP e TCP e os protocolos de roteamento AODV, AOMDV, DSR e DSDV. O modelo de propagação de rádio é o Two Rayground, enquanto a camada MAC segue as especificações IEEE 802.11p [18]. As simulações são realizadas por 600 segundos e todos os resultados apresentados são médias de 35 simulações com o mesmo modelo de tráfego, mas com diferentes cenários de mobilidade. Para estas simulações o intervalo de confiança considerado é de $95 \%$. Os parâmetros comuns para todas as simulações realizadas estão resumidos na Tabela I.

Tabela I: Parâmetros comuns para todas as simulações realizadas.

\begin{tabular}{|c|c|c|}
\hline \multirow{2}{*}{ Parâmetros } & \multicolumn{2}{|c|}{ Valores } \\
\cline { 2 - 3 } & TCP/CBR & UDP/CBR \\
\hline Tipo de Tráfego & CBR & CBR \\
\hline Protocolo de transporte & TCP & UDP \\
\hline Modelo de propagação & Two Rayground & Two Rayground \\
\hline Tamanho do Cenário & $1000 x 1000$ (metros) & $1000 x 1000$ (metros) \\
\hline Protocolos de roteamento & AODV, AOMDV, & AODV, AOMDV, \\
\hline Camada MSDV & DSDV \\
\hline Raio de alcance & IEEE $802.11 \mathrm{p}$ & IEEE 802.11p \\
\hline Conexões simultâneas & $250 \mathrm{~m}$ & $250 \mathrm{~m}$ \\
\hline Tempo de Simulação & 5 & $500 \mathrm{~s}$ \\
\hline
\end{tabular}

\section{Modelos de simulação}

Neste trabalho são utilizados quatro modelos de simulações com diferentes condições adversas. 
1) Modelo de Simulação 1 (variando a velocidade): neste modelo, os veículos possuem velocidades extremas que variam entre $60 \mathrm{~km} / \mathrm{h}$ e $150 \mathrm{~km} / \mathrm{h}$. Tais velocidades representam condições adversas reais que poderão ocorrer (por exemplo: ambientes rápidos em pistas que permitem altas velocidades). A Tabela II mostra os parâmetros utilizados especificamente no Modelo de Simulação 1.

Tabela II: Parâmetros do Modelo de Simulação 1.

\begin{tabular}{|c|c|c|}
\hline \multirow{2}{*}{ Parâmetros } & \multicolumn{2}{|c|}{ Valores } \\
\cline { 2 - 3 } & TCP/CBR & UDP/CBR \\
\hline Número de Nós & 75 & 75 \\
\hline Mensagens por Segundo & 4 & 4 \\
\hline Velocidade dos Nós & $60,90,120,150 \mathrm{~km} / \mathrm{h}$ & $60,90,120,150 \mathrm{~km} / \mathrm{h}$ \\
\hline Tamanho da Mensagem & 512 bytes & 512 bytes \\
\hline
\end{tabular}

2) Modelo de Simulação 2 (variando a densidade): neste modelo, os veículos possuem densidades extremas (entre $100 \mathrm{e}$ 400 veículos na rede). Tais densidades representam condições adversas reais que poderão ocorrer (por exemplo: ambientes urbanos em horário de pico ou em eventos). A Tabela III mostra os parâmetros utilizados especificamente no Modelo de Simulação 2.

Tabela III: Parâmetros do Modelo de Simulação 2.

\begin{tabular}{|c|c|c|}
\hline \multirow{2}{*}{ Parâmetros } & \multicolumn{2}{|c|}{ Valores } \\
\cline { 2 - 3 } & TCP/CBR & UDP/CBR \\
\hline Número de Nós & $100,200,300,400$ & $100,200,300,400$ \\
\hline Mensagens por Segundo & 4 & 4 \\
\hline Velocidade dos Nós & $50 \mathrm{~km} / \mathrm{h}$ & $50 \mathrm{~km} / \mathrm{h}$ \\
\hline Tamanho da Mensagem & $512 \mathrm{bytes}$ & $512 \mathrm{bytes}$ \\
\hline
\end{tabular}

3) Modelo de Simulação 3 (variando a taxa de dados): neste modelo, os veículos transmitem taxas de dados extremas. Neste cenário, os veículos estão inseridos em ambientes que enviam entre 32 e 256 mensagens de 512 bytes por segundo, ou seja, entre $16 \mathrm{kbytes} / \mathrm{s}$ e $132 \mathrm{kbytes} / \mathrm{s}$. O cenário descrito representa condições adversas reais que poderão ocorrer (por exemplo: transmissão rápida de dados armazenados em pontos fixos da rodovia). A Tabela IV mostra os parâmetros utilizados especificamente no Modelo de Simulação 3.

Tabela IV: Parâmetros do Modelo de Simulação 3.

\begin{tabular}{|c|c|c|}
\hline \multirow{2}{*}{ Parâmetros } & \multicolumn{2}{|c|}{ Valores } \\
\cline { 2 - 3 } & TCP/CBR & UDP/CBR \\
\hline Número de Nós & 75 & 75 \\
\hline Velocidade dos Nós & $50 \mathrm{~km} / \mathrm{h}$ & $50 \mathrm{~km} / \mathrm{h}$ \\
\hline Mensagens por Segundo & $32,64,128 \mathrm{e} 256$ & $32,64,128 \mathrm{e} 256$ \\
\hline Tamanho da Mensagem & 512 bytes & 512 bytes \\
\hline
\end{tabular}

4) Modelo de Simulação 4 (variando o volume dos dados): neste modelo, os veículos transmitem volumes de dados extremos (mensagens com tamanhos que variam de 768 bytes e 4096 bytes). O cenário descrito também representa condições adversas reais que poderão ocorrer (com o aumento do número de aplicações que farão parte das comunicações veiculares, os veículos necessitarão transmitir dados com os mais variados tamanhos para outros veículos e pontos fixos). A Tabela V mostra os parâmetros utilizados especificamente no Modelo de Simulação 4.

Tabela V: Parâmetros do Modelo de Simulação 4.

\begin{tabular}{|c|c|c|}
\hline \multirow{2}{*}{ Parâmetros } & \multicolumn{2}{|c|}{ Valores } \\
\cline { 2 - 3 } & TCP/CBR & UDP/CBR \\
\hline Número de Nós & 75 & 75 \\
\hline Velocidade dos Nós & $50 \mathrm{~km} / \mathrm{h}$ & $50 \mathrm{~km} / \mathrm{h}$ \\
\hline $\begin{array}{c}\text { Mensagens por } \\
\text { Segundo }\end{array}$ & 4 & 4 \\
\hline $\begin{array}{c}\text { Tamanho da } \\
\text { Mensagem }\end{array}$ & $768,1024,2048,4096$ bytes & $768,1024,2048 \mathrm{e}$ \\
4096 bytes \\
\hline
\end{tabular}

\section{Resultados E Discussão}

Nesta seção serão apresentados os resultados das simulações. Os gráficos apresentam no eixo " $x$ " as variações dos parâmetros que tornam os cenários adversos (densidade, velocidade, taxa e volume de dados), e no eixo "y" os valores obtidos para a métrica de desempenho (taxa de entrega).

\section{A. Modelo de Simulação 1}

A Figura 1 apresenta os resultados da taxa de entrega de pacotes em função da variação da velocidade para o Modelo de Simulação 1 com o protocolo UDP. É possível notar que o pior desempenho apresentado foi do protocolo proativo DSDV. O AODV; DSR; AOMDV (nesta ordem) foram os que apresentaram os melhores resultados. Isso ocorre pela boa adaptação do processo de manutenção de rotas de protocolos de roteamento reativos a cenários altamente dinâmicos com o protocolo de transporte UDP.

A Figura 2 apresenta os resultados da taxa de entrega de pacotes em função da variação da velocidade para o Modelo de Simulação $1 \mathrm{com}$ o protocolo TCP. É possível notar que o protocolo com melhor taxa de entrega é o DSR. O DSR, com seu processo eficiente de manutenção de rotas, foi o que teve melhor adaptação ao uso do protocolo de transporte TCP em cenários altamente dinâmicos (com frequentes quebras de rotas devido a elevada velocidade dos nós). Na sequência, o DSDV (protocolo proativo) mostrou sua eficiência em manter as rotas sempre atualizadas em ambientes orientados a conexão, superando o restante dos protocolos reativos (AODV e AOMDV). Além disso, devido ao TCP oferecer um ambiente de transmissões confiável, com mecanismos de correção de erros, a taxa de entrega foi sempre melhor quando comparada a ambientes que utilizam o protocolo UDP (Fig. 1).

\section{B. Modelo de Simulação 2}

A Figura 3 mostra os resultados da taxa de entrega de pacotes em função da variação do número de veículos para o Modelo de Simulação 2 com o protocolo UDP. Nota-se que com o aumento extremo do número de veículos os protocolos reativos obtiveram os melhores resultados, com destaque para o AODV, o qual manteve a taxa de entrega entre $80 \%$ e $90 \%$ 
mesmo com 400 nós na rede. Já o protocolo DSDV (pró-ativo) não conseguiu executar suas operações a partir de 300 veículos na rede.

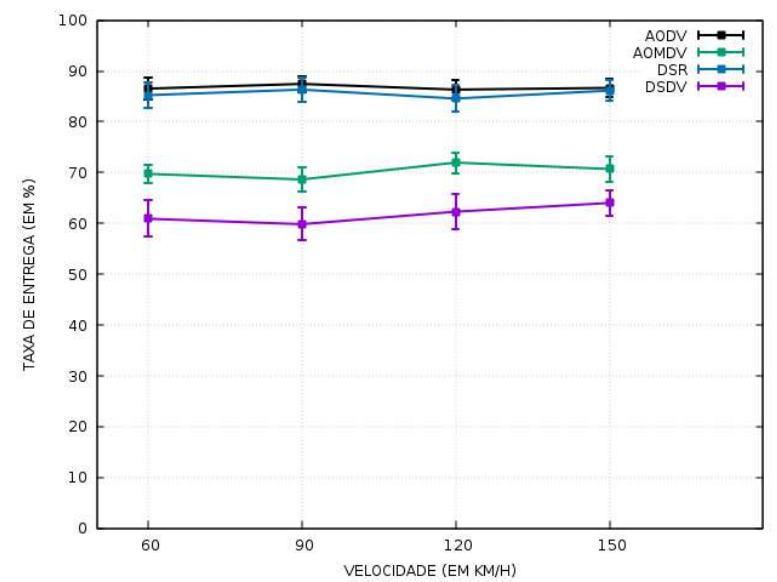

Fig 1: TE com UDP para o Modelo de Simulação 1.

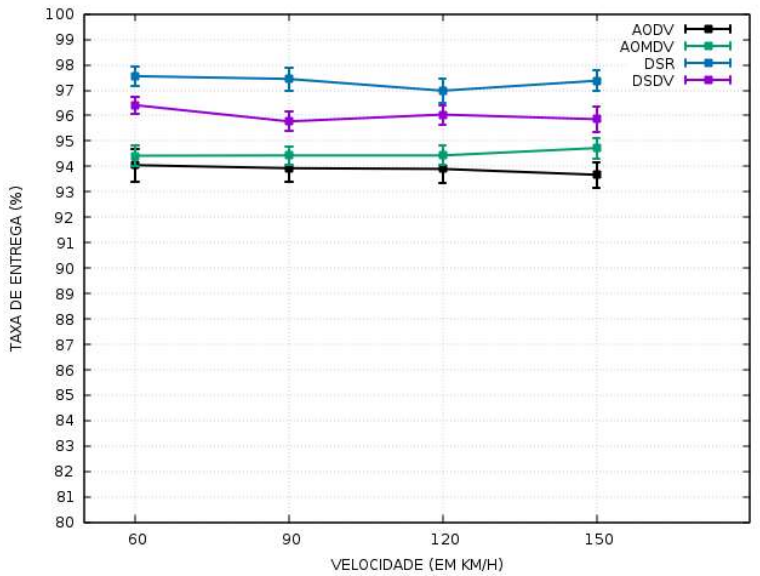

Fig 2: TE com TCP para o Modelo de Simulação 1.

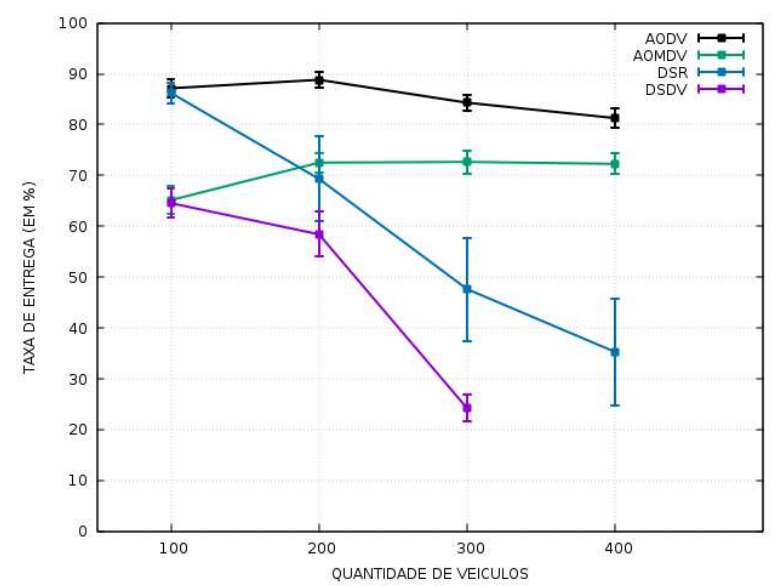

Fig 3: TE com UDP para o Modelo de Simulação 2.

A Figura 4 mostra os resultados da taxa de entrega de pacotes em função da variação do número de veículos para o Modelo de Simulação 2 com o protocolo TCP. Nota-se que todos os protocolos reativos tiveram bom desempenho (com destaque para o DSR), mantendo a taxa de entrega maior do que $90 \%$ em todos os casos. Já o protocolo DSDV (reativo), apresentou desempenho satisfatório com até 200 veículos (taxa de entrega maior do que $95 \%$ ), degradando-se na sequência, até não conseguir mais operar a partir de 300 veículos (mesmo com o TCP como protocolo de transporte).

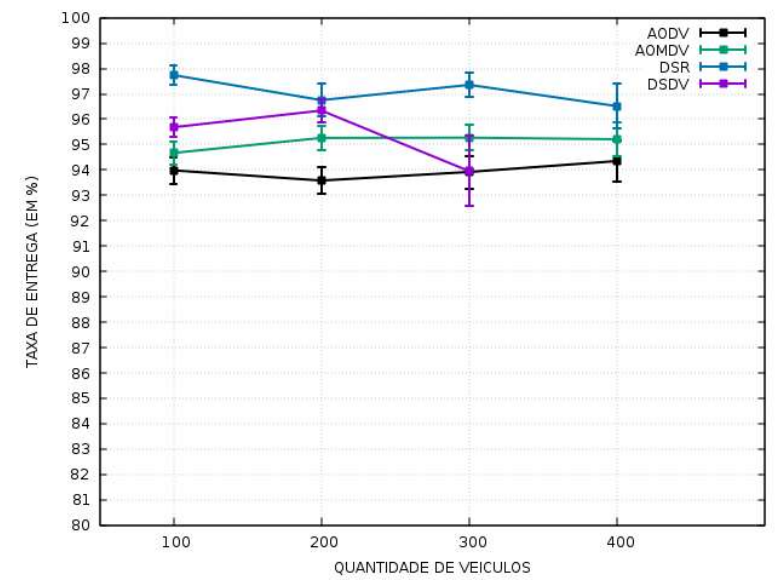

Fig 4: TE com TCP para o Modelo de Simulação 2.

\section{Modelo de Simulação 3}

A Figura 5 mostra os resultados da taxa de entrega de pacotes em função da taxa de dados transferidos (número de mensagens por segundo) para o Modelo de Simulação 3 com o protocolo UDP. Nota-se que todos os protocolos tiverem desempenho semelhante quanto a taxa de dados transferidos. Em todos os casos a taxa de entrega reduziu com o aumento do número de mensagens enviadas por segundo (não ultrapassando $50 \%)$.

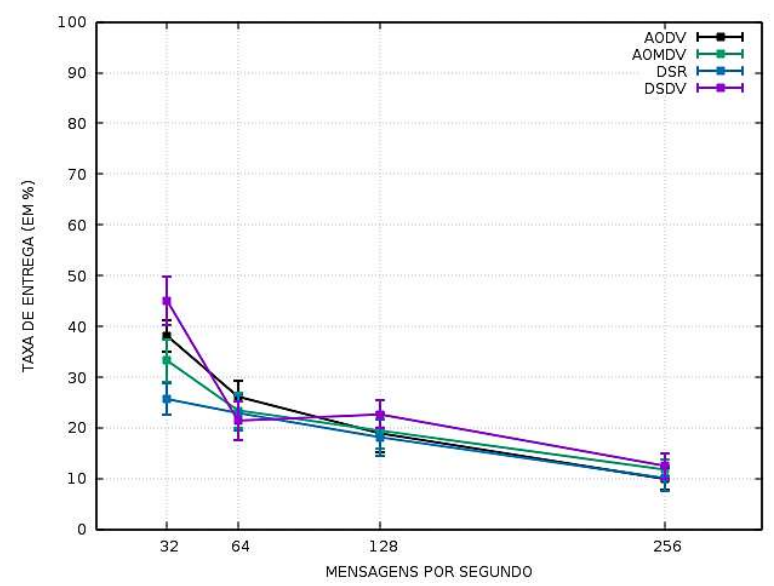

Fig. 5: TE com UDP para o Modelo de Simulação 3.

A Figura 6 mostra os resultados da taxa de entrega de pacotes em função da taxa de dados para o Modelo de Simulação $3 \mathrm{com}$ o protocolo TCP. Nota-se que todos os protocolos tiverem desempenhos semelhantes (com destaque para o protocolo DSR). Em todos os casos, com o uso do TCP, o desempenho manteve-se estável com o aumento do número de mensagens enviadas por segundo, e sempre superior a $97 \%$ (situação não observada com o UDP (Fig. 5)).

\section{Modelo de Simulação 4}

A Figura 7 mostra os resultados da taxa de entrega de pacotes em função da variação do volume dos dados (tamanho da mensagens) para o Modelo de Simulação 4 com o protocolo UDP. Observa-se que o aumento do tamanho das mensagens implica na redução do desempenho de todos os protocolos (mesmo assim o AODV apresentou os melhores resultados). Isso ocorre porque quanto maior o tamanho das mensagens, maior será o tempo em que os veículos necessitarão estar conectados para concluir a transmissão. Entretanto, com a natureza dinâmica da rede, os veículos não conseguem ficar conectados por um longo período de tempo, aumentando a quantidade de pacotes perdidos (agravando-se ainda mais 
devido ao UDP não ter nenhum mecanismo de retransmissão e garantia de entrega).

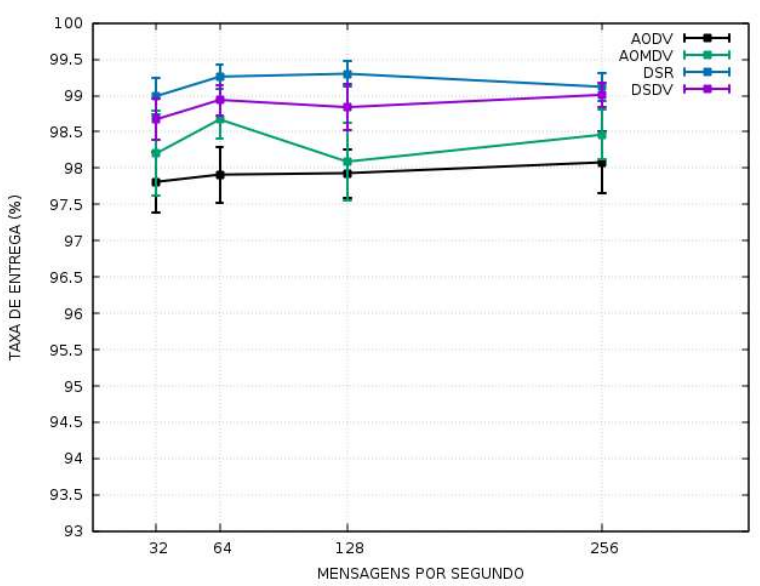

Fig. 6: TE com TCP para o Modelo de Simulação 3.

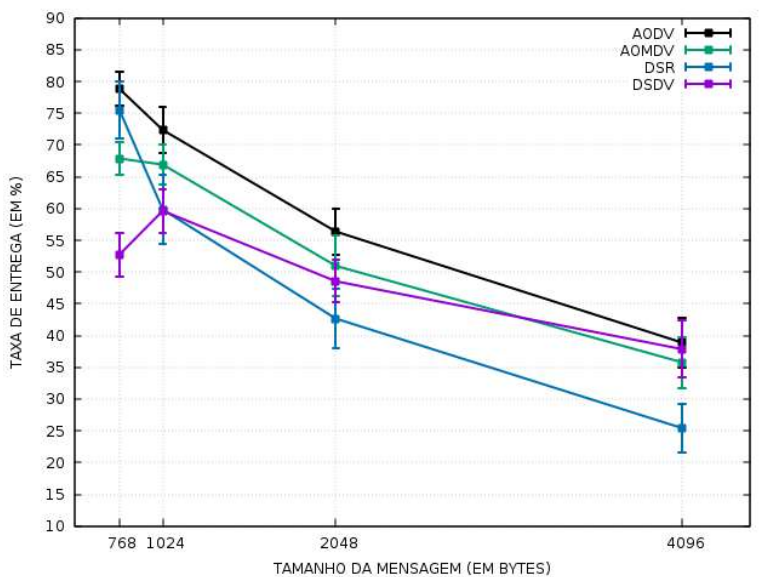

Fig. 7: TE com UDP para o Modelo de Simulação 4.

A Figura 8 mostra os resultados da taxa de entrega de pacotes em função da variação do volume dos dados para o Modelo de Simulação 4 com o protocolo TCP. Nota-se na que todos os protocolos tiverem desempenhos semelhantes (com destaque para o protocolo DSR). Em todos os casos, com o uso do TCP, o desempenho manteve-se estável com o aumento do tamanho das mensagens e sempre superior a 93\% (situação não observada com o UDP (Fig. 7)). Isso ocorre pois no TCP, independentemente do tamanho da mensagem enviada, as mesmas são fragmentadas em tamanhos fixos (evitando assim o impacto de lidar com pacotes de tamanhos excessivamente grandes)

\section{CONCLUSÃO}

Este trabalho apresentou avaliações de desempenho, por meio de simulações, em redes veiculares com condições adversas. Resultados de simulação demonstraram que o protocolo de roteamento AODV apresentou os melhores resultados entre todos os protocolos avaliados atuando em conjunto com o UDP. Quando o TCP esteve presente na rede, o protocolo DSR apresentou os melhores resultados. Além disso, o TCP por oferecer um ambiente confiável (com estabelecimento de conexão, retransmissões, controle de fluxo e congestionamento), apresentou desempenho sempre superior ao UDP. Trabalhos futuros devem incluir a análise de novas métricas de desempenho, como o atraso, a vazão e a sobrecarga de roteamento.

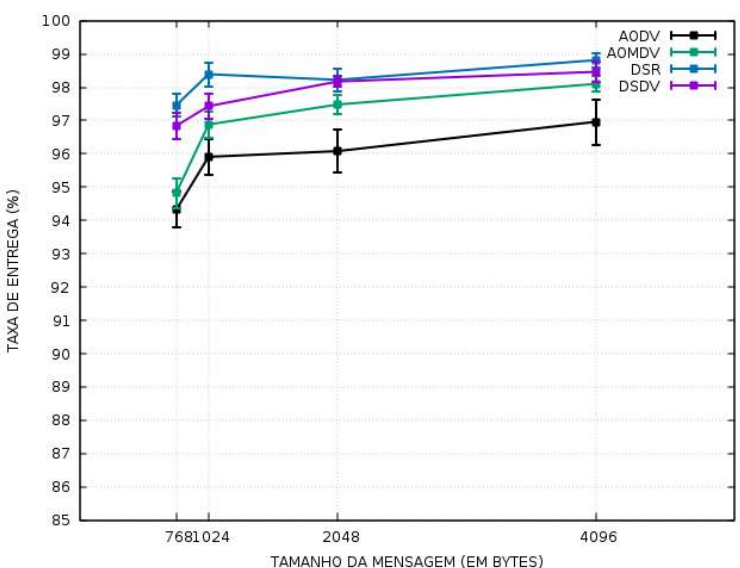

Fig. 8: TE com TCP para o Modelo de Simulação 4.

\section{REFERENCES}

[1] BENZA, M.; BERSANI, C Intelligent Transport Systems (ITS) applications on dangerous good transport on road in Italy. In: SoSE, 2012.

[2] ALVES JR, Joilson; WILLE, Emilio. Improving VANETs Connectivity with a Totally Ad Hoc Living Mobile Backbone. Journal of Computer Networks and Communications. Hindawi. 2015.

[3] ALVES JR, Joilson; WILLE, Emilio. P-AOMDV: An improved routing protocol for $\mathrm{V} 2 \mathrm{~V}$ communication based on public transport backbones. Trans. on Emerg. Telecom. Technologies. 2016.

[4] SANGUESA, Julio A. Topology-based Broadcast Schemes for Urban Scenarios Targeting Adverse Density Conditions. In: Wireless Communications and Networking Conference (WCNC). IEEE, 2014.

[5] MARTIN-FERNÁNDEZ, F.; CABALlERO-GIL, P.; CABALLEROGIL, C. An Experimental Hybrid Wireless Platform for Vehicula Networks. In: A World of Wireless, Mobile and Multimedia Networks (WoWMoM), 2016 IEEE 17th Int. Symposium on. IEEE, 2016.

[6] PERKINS, Charles E.; ROYER, E. M. Ad-hoc on-demand distance vector routing. In: Proceedings of the Second IEEE Workshop on Mobile Computer Systems and Applications. IEEE, 1999.

[7] MARINA, Mahesh K.; DAS, Samir R. Ad hoc on-demand multipath distance vector routing. SIGMOBILE Mob. Comput. Commun., 2002.

[8] JOHNSON, David B.; MALTZ, David A. Dynamic source routing in ad hoc wireless networks. In: Imielinski T., Korth H.F. (eds) Mobile Comp. The Kluwer Int. Series in Engineering and Computer Science, 1996.

[9] PERKINS, Charles E.; BHAGWAT, Pravin. Highly dynamic destinationsequenced distance-vector routing (DSDV) for mobile computers. SIGCOMM Comput. Commun, 1994.

[10] FOROUZAN, Behrouz A. Comunicação de dados e redes de computadores. 4. ed. - Dados eletrônicos. - Porto Alegre: AMGH, 2010.

[11] SALLUM, Eduardo El Akkari. Performance analysis and comparison of the DSDV, AODV and OLSR routing protocols under VANETs. In: ITST, 2018.

[12] KANG, Seung-Seok; CHAE, Ye-Eun; YEON, Seunguk. VANET Routing Algorithm Performance Comparison using NS-3 and SUMO. In Computer Applications and Information Processing Technology (CAIPT), 2017

[13] SHARMA, Harshit; T., Jayavignesh. Comparative Analysis and Applicability of Routing Protocols in VANETs. In: Electrical, Electronics, Communication, Computer, and Optimization Techniques (ICEECCOT), 2017.

[14] KUMAR, Vimlesh; BAGHEL, Anurag Singh; MISHRA, Priyank. Performance Evaluation of DSDV, AODV and LSGR Protocol in Ad Hoc Networks. In: ICEEOT, 2016

[15] NS2. The network simulator. 2019. Disponível em https://www.isi.edu/nsnam/ns/index.html. Acesso em: 18 mar. 2019.

[16] VANETMOBISIM. VanetMobiSim. 2019. Disponível em: http://vanet.eurecom.fr. Acesso em: 15 mar. 2019.

[17] PAUL, Bijan; IBRAHIM, Md.; BIKAS, Md. Abu Naser. Experimental Analysis of AODV \& DSR over TCP \& CBR Connections with Varying Speed and Node Density in VANET. Int. J. of Comp. Applications, 2011.

[18] IEEE. Draft Amendment for Wireless Access in Vehicular Environments (WAVE), IEEE Std. P802.11p / D3.0, Julho, 2007. 